\title{
Post-Procedural Evaluation of Catheter Contact Force Characteristics
}

\author{
Martin $\operatorname{Koch}^{a}$, Alexander Brost ${ }^{a}$, Atilla Kiraly ${ }^{b}$, Norbert Strobel $^{c}$, Joachim Hornegger ${ }^{a, d}$ \\ ${ }^{a}$ Pattern Recognition Lab, Friedrich-Alexander-University \\ Erlangen-Nuremberg, Erlangen, Germany \\ ${ }^{b}$ Siemens Corporate Research, Princeton, NJ, USA \\ ${ }^{c}$ Siemens AG, Healthcare Sector, Forchheim, Germany \\ ${ }^{d}$ School in Advanced Optical Technologies (SAOT), Erlangen, Germany
}

\begin{abstract}
Minimally invasive catheter ablation of electric foci, performed in electrophysiology labs, is an attractive treatment option for atrial fibrillation (AF) - in particular if drug therapy is no longer effective or tolerated. There are different strategies to eliminate the electric foci inducing the arrhythmia. Independent of the particular strategy, it is essential to place transmural lesions. The impact of catheter contact force on the generated lesion quality has been investigated recently, and first results are promising. There are different approaches to measure catheter-tissue contact. Besides traditional haptic feedback, there are new technologies either relying on catheter tip-to-tissue contact force or on local impedance measurements at the tip of the catheter.

In this paper, we present a novel tool for post-procedural ablation point evaluation and visualization of contact force characteristics. Our method is based on localizing ablation points set during AF ablation procedures. The 3 -D point positions are stored together with lesion specific catheter contact force $(\mathrm{CF})$ values recorded during the ablation. The force records are mapped to the spatial 3-D positions, where the energy has been applied. The tracked positions of the ablation points can be further used to generate a 3-D mesh model of the left atrium (LA). Since our approach facilitates visualization of different force characteristics for post-procedural evaluation and verification, it has the potential to improve outcome by highlighting areas where lesion quality may be less than desired.
\end{abstract}

Keywords: catheter contact force, atrial fibrillation, radiofrequency ablation, electrophysiology

\section{MOTIVATION}

For treatment of heart arrhythmias such as AF, minimally invasive catheter ablations are one method of choice. ${ }^{1}$ Ablation lesions are usually generated at certain areas inside the left atrium. The correct positions are determined by intracardiac electrocardiograms and the chosen ablation strategy. A common strategy for treatment of paroxysmal AF is pulmonary vein isolation (PVI). ${ }^{2}$ After PVI, electrical signals originating from the pulmonary veins are blocked from entering the LA. This can be achieved by placing ablation lines around the four pulmonary vein ostia. During PVI procedures, the quality of the generated ablation points is crucial. Lesions need to be transmural to be permanent. If lesions are not transmural, they may recover over time, and the newly formed tissue may become electrically conductive again. In those cases, an additional ablation procedure is often necessary.

The importance of catheter contact force measurement has been shown in recent studies, ${ }^{3,4}$ correlating contact force patterns with treatment outcome for paroxysmal atrial fibrillation. New technologies for measuring the catheter tissue contact, based on tip-to-tissue contact force, ${ }^{5}$ or local impedance measurements at the tip of the catheter ${ }^{6}$ have been introduced.

In the following section, we describe our approach to record the applied contact force during the course of ablation together with the spatial distribution of ablation points.

Send correspondence to Martin.Koch@cs.fau.de 


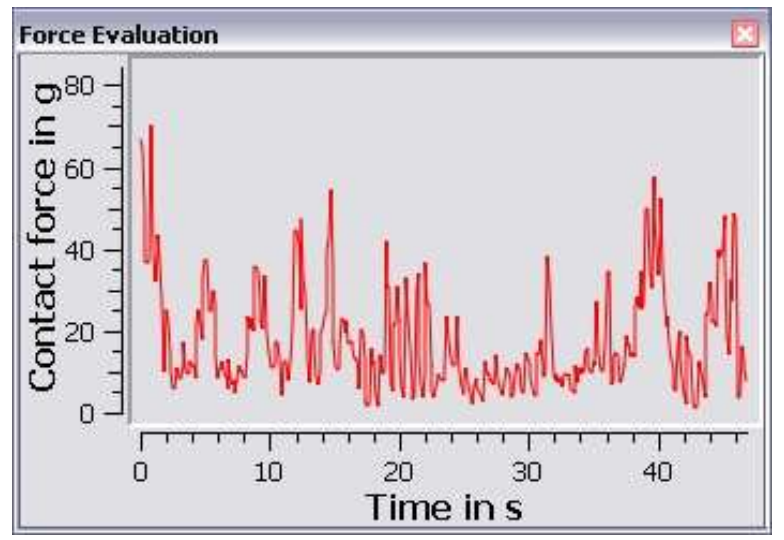

(a)

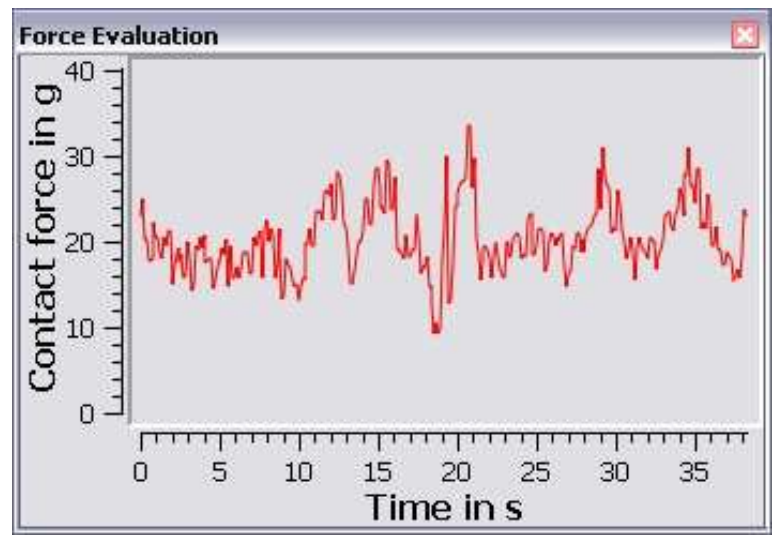

(c)

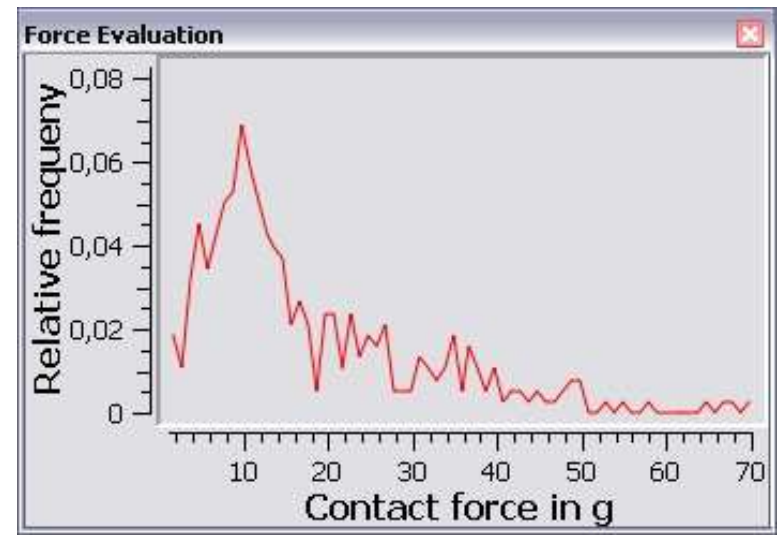

(b)

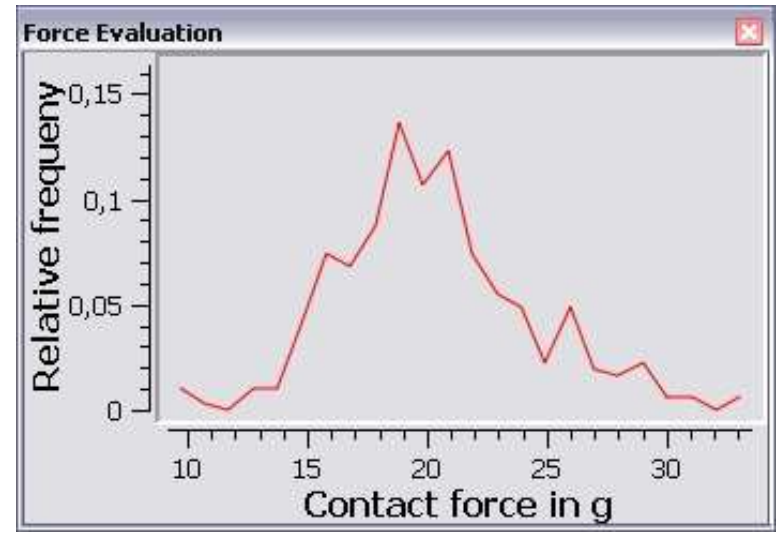

(d)

Figure 1. Contact force graph for the duration of two ablation points (a), (c). Histogram plot of relative frequencies (b), (d).

\section{METHOD}

During the course of ablation, the force measurements are processed in real-time, and stored for later evaluations. ${ }^{7}$ For each ablation point, a vector of discrete samples of the continuous contact force signal is recorded. In addition, the 3-D position of each ablation point is tracked. This is achieved via triangulation of the ablation catheter tip from two perpendicular X-ray views using a bi-plane X-ray imaging system. ${ }^{8}$ Based on the combination of spatial and functional data, ablation point specific evaluations can be performed.

\subsection{Force Characteristics}

Even though the importance of catheter contact force is widely accepted, no consensus has been established yet which parameters are most important and what their proper values should be. For this reason, we wanted to provide a platform to test and evaluate current contact force characteristics and allow for new concepts as well. To this end, stored contact force samples are processed ablation point wise.

In Figure 1 the contact force of single ablation points are shown over time. The graph nicely shows the high variability in catheter tip-to-tissue contact during the ablation. The composition of the force signal is analyzed in Figures 1(b) and (d). The histogram for each ablation point is shown in terms of relative frequencies per force value. Contact force values are distributed around $10 \mathrm{~g}$ in the first example and around $20 \mathrm{~g}$ for the second, respectively. 


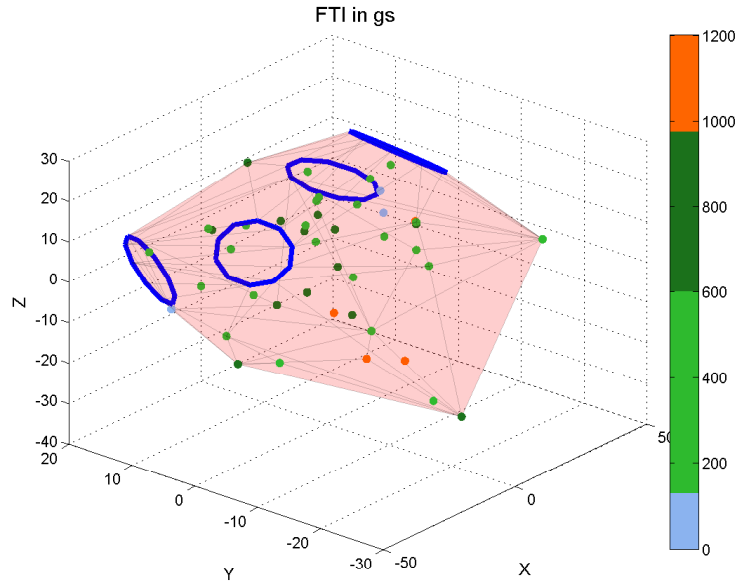

(a)

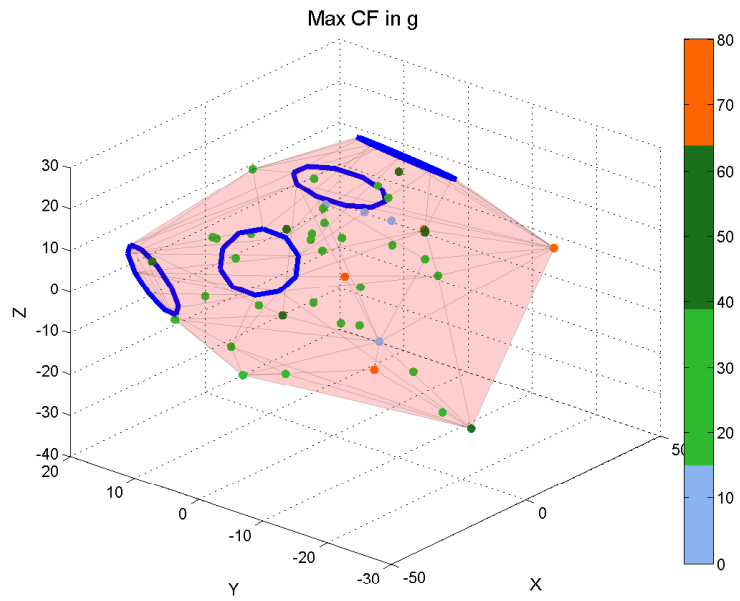

(c)

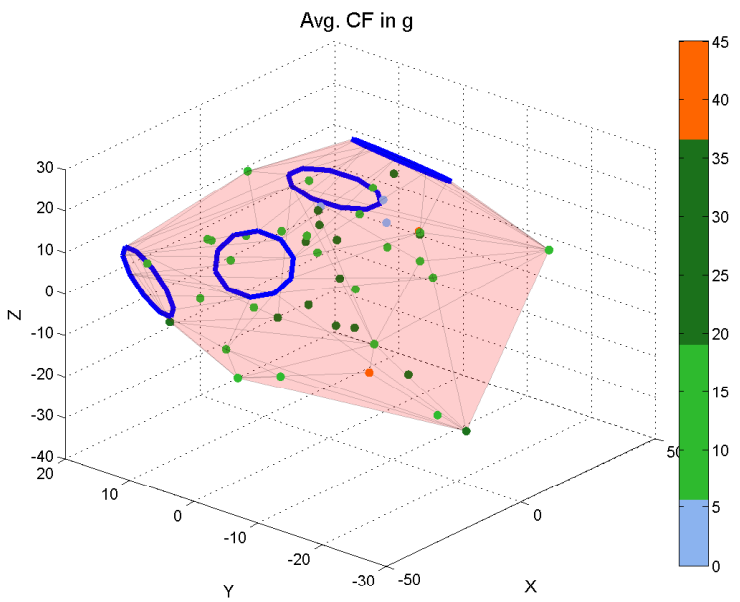

(b)

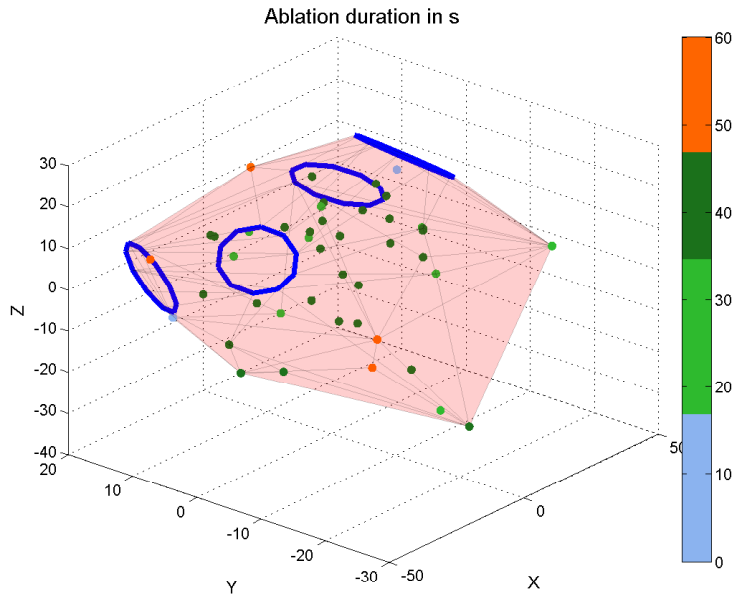

(d)

Figure 2. Ablation points can be shown with different colors to highlight various force features such as, e.g., forcetime-integral, average force, and maximum force, respectively. The loop-like structures represent pulmonary vein (PV) ostia. These structures were acquired by $3-\mathrm{D}$ reconstruction of a circumferential mapping catheter from two views. (a) Aggregated force values as force-time-integral (FTI) in gs. (b) Average catheter contact force in g. (c) Maximal catheter contact force in $\mathrm{g}$. (d) Representation of the ablation duration per point in seconds.

\subsection{Features}

There are several possible force parameters one can consider for evaluation, such as average contact force, minimum contact force, or maximum contact force, respectively. There are also derived parameters such as the force-time-integral (FTI). ${ }^{9}$ The FTI may be a very good parameter for lesion quality assessment, but more research is needed to verify this hypothesis.

Thinking ahead, other features, not directly related to contact force, yet potentially correlated to lesion quality, can be evaluated as well. These parameters could be the power of the radio-frequency (RF) generator, resulting current, the temperature at the tip of the catheter or even features extracted from other imaging modalities such as ultrasound ${ }^{10}$ or late enhanced MR images. ${ }^{11}$

\subsection{Visualization}

The visualization framework supports setting of thresholds to color code ablation points according to their force characteristics. The thresholds can be set, for example, to separate the collected data into three main clusters 


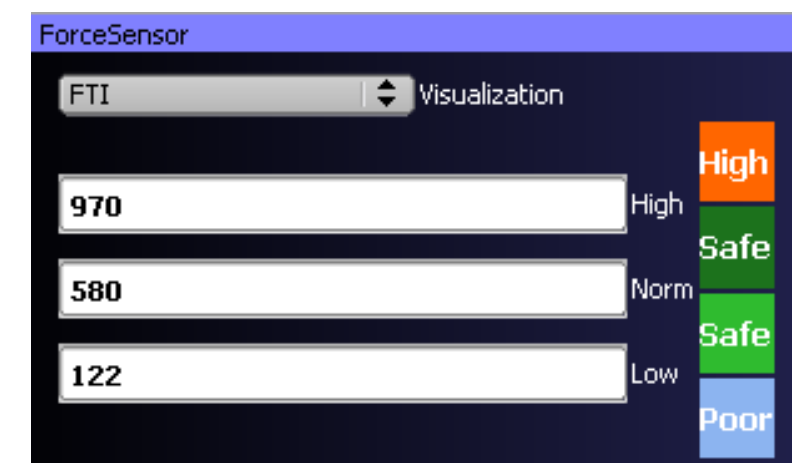

Figure 3. Interface to interactively set threshold parameters.

\begin{tabular}{|c|c|c|c|}
\hline CF parameter & 'Poor' & 'Safe' & 'High' \\
\hline FTI & 0.09 & 0.84 & 0.07 \\
\hline Avg CF & 0.07 & 0.87 & 0.06 \\
\hline Duration & 0.08 & 0.87 & 0.05 \\
\hline Std. Dev. & 0.07 & 0.87 & 0.07 \\
\hline Max CF & 0.04 & 0.88 & 0.08 \\
\hline
\end{tabular}

Table 1. Classification of clinical contact force data based on estimated thresholds.

labeled poor, safe and high. The middle cluster ("safe") can be further subdivided into below- and above average, specified by the norm threshold. This value represents the expected mean value of the data distribution. The interface to set those values is shown in Figure 3.

\subsection{Delaunay triangulation}

Ablation points are detected on the myocardium of the left atrium and localized in 3-D from two views, e.g., using a bi-plane $\mathrm{C}$-arm system. If these points are taken during ablation, they provide a rough estimate of the LA outline. The collected point cloud is used to reconstruct a model. More specifically, a delaunay triangulation is applied to generate a triangulation of the convex hull of the ablation points. Based on this structure, a surface mesh can be created.

\section{EVALUATION AND RESULTS}

To determine reasonable values for the classification thresholds, data collected during atrial fibrilation catheter ablation procedures have been evaluated. Catheter contact force data has been recorded for a total of 209 ablation lesions from five different patients. Based on this data pool, the mean value as well as 10 and 90 percentile have been calculated for each of the force parameters. The box-plots for these parameters are shown in Figure 4.

These classification threshold settings have been applied to new clinical data from three patients received during atrial fibrilation ablation procedures. In Table 1 the classification results of the ablation points are shown. Note that more clinical insight is needed before these classification thresholds can be relied on. There are clinical studies ongoing investigating force parameters that significantly effect lesion quality and thereby treatment outcome. ${ }^{12}$

To get an idea if thresholds derived for a number of cases carry over to new cases, we analyzed the distribution of the FTI parameter more closely. In Figure 5(a), a Gaussian and a Rayleigh distribution are shown along with the histogram of the FTI values comprising five cases. The respective distributions have been fitted to the underlying training data. According to our data, the Rayleigh distribution seems to models the distribution of the FTI values more accurately than the Gaussian distribution. Especially for low FTI values, the Rayleigh distribution provides a better estimate. It also satisfies the constraint, that there cannot be an ablation point with FTI smaller or equal to 0. In Figure 5(b), the blue and red curves represent the distribution of the FTI parameter for each of our eight patients. The thick black line represents the collection of data used for estimating the classification thresholds (first five patients). We find that the Rayleigh distributions of the different cases all 


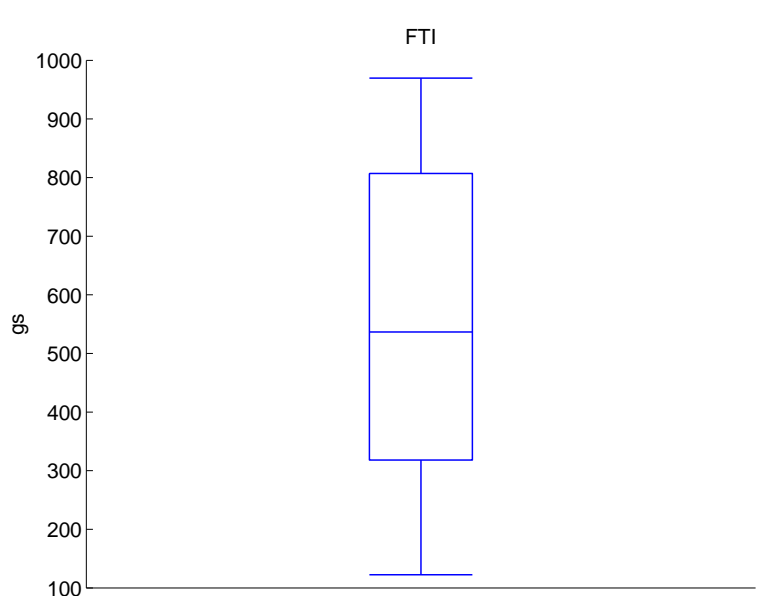

(a)

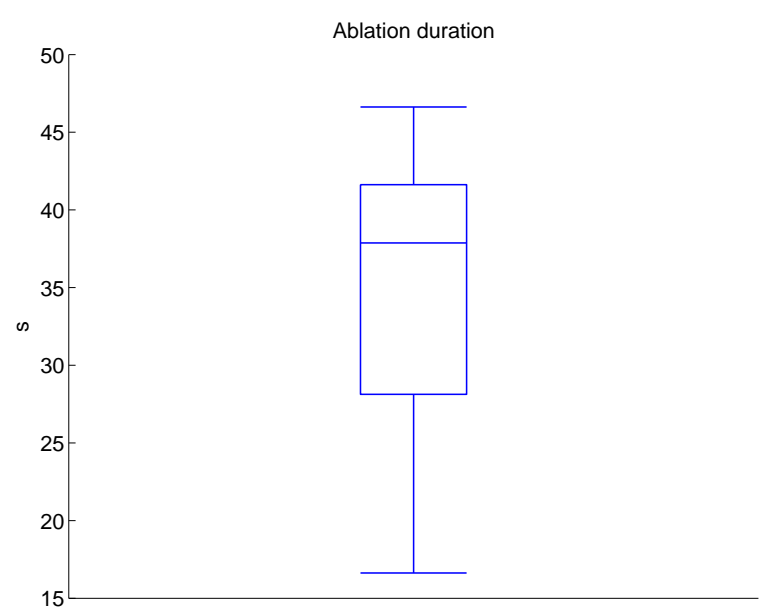

(c)

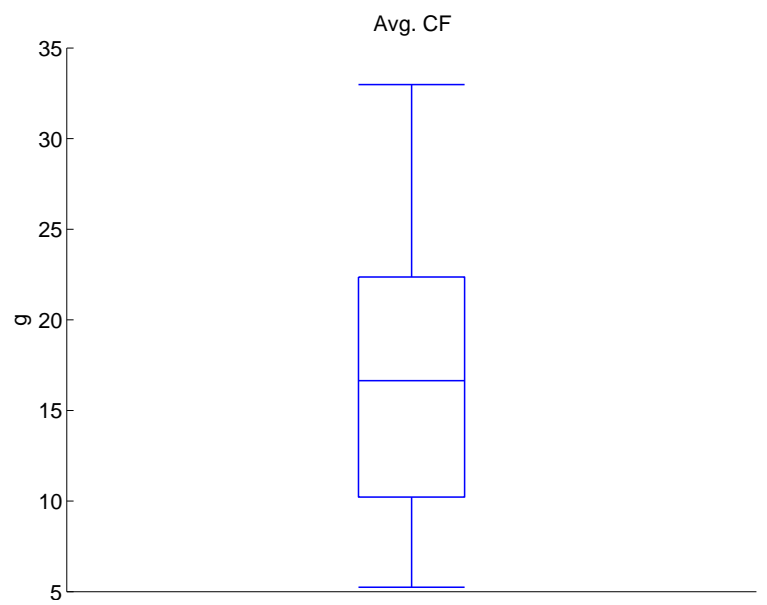

(b)

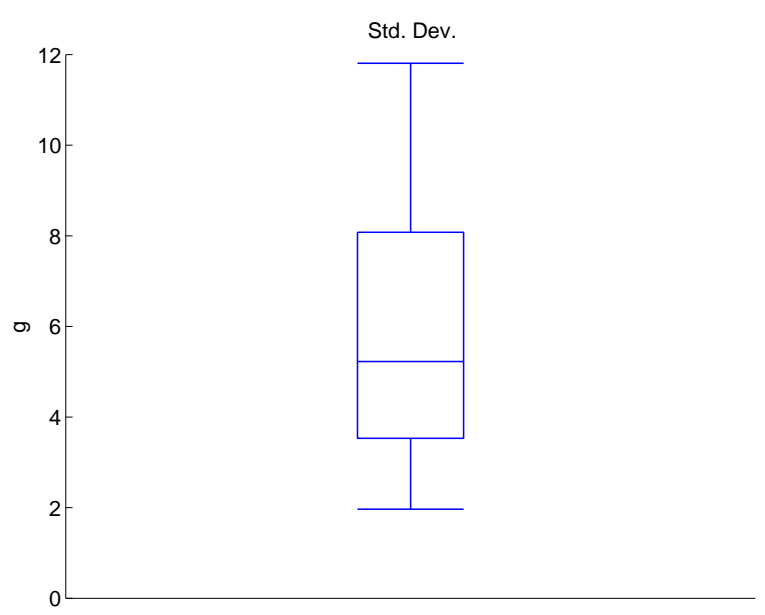

(d)

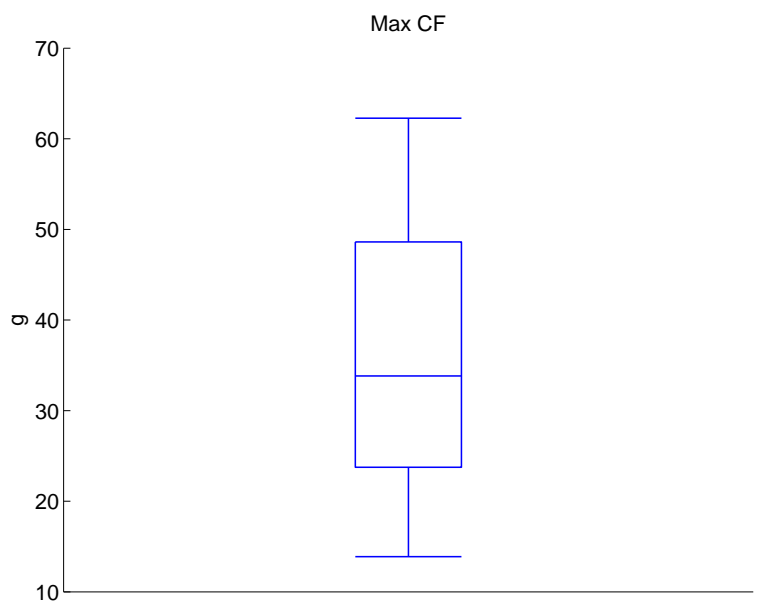

(e)

Figure 4. Box-plots for selected contact force characteristics based on 209 ablation points from five different patients. 


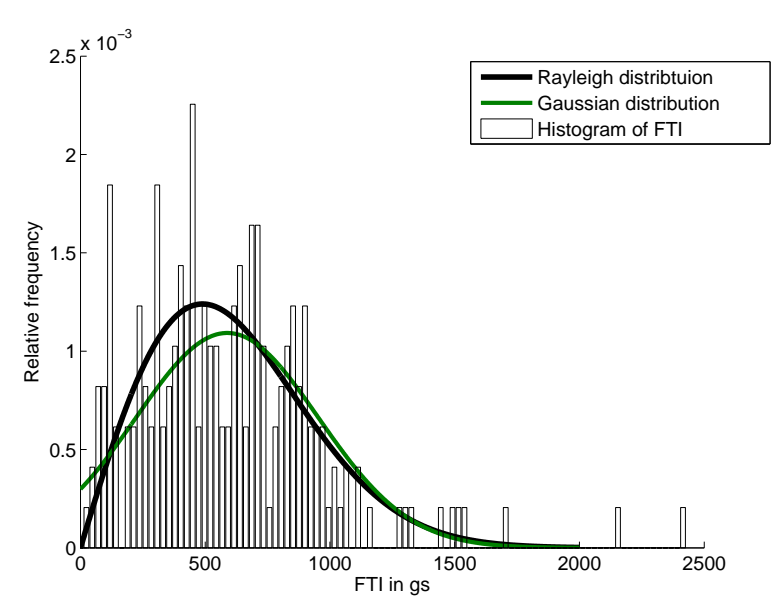

(a)

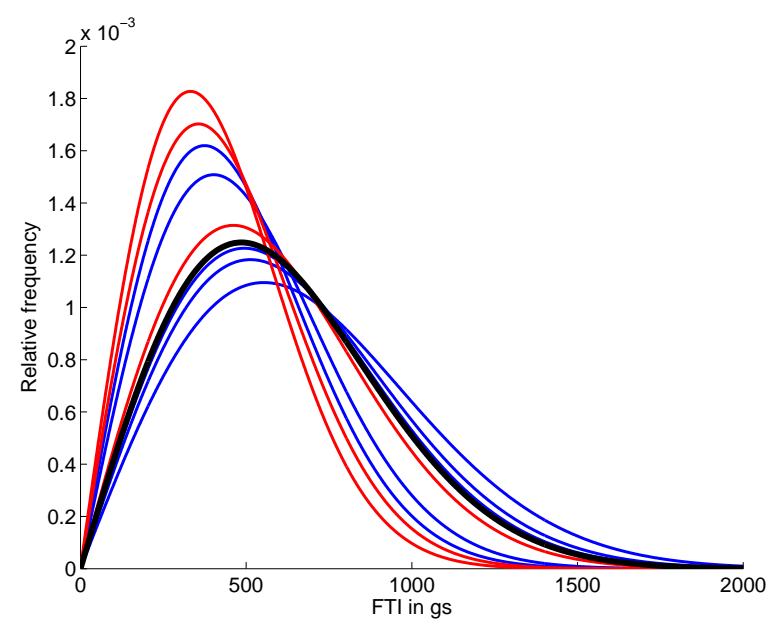

(b)

Figure 5. Distribution of the FTI values. (a) Histogram of the FTI values for first five patients together with estimated Gaussian and Rayleigh distributions. (b) Rayleigh distributions of the FTI for different procedures. The blue curves represent data used for calculating the classification thresholds, whereas the red curves show additional procedure data. The black line visualizes a Rayleigh distribution based on training data comprising all FTI values of the first five patients.

cluster around a common global mean. This justifies our approach of applying classification thresholds calculated based on a certain data pool to new unseen data sets. Even though slight modification on a case by case basis might be necessary, a generally valid initialization appears feasible.

There are different options for visualizations of this data. In Figure 2 different parameters and contact force characteristics for an ablation procedure are shown. In this scenario the convex hull of the tracked ablation points is used to compute an estimate of the object's inner surface and is visualized as a mesh structure. The mesh facilitates the perception of 3-D information and spatial orientation. In this case, FTI, average and maximal force values, as well as ablation duration are shown. If patient specific 3-D volume data sets are available, an overlay of the actual heart model is feasible as well. In Figure 6(a), the ablation points are shown on a 3-D left atrium mesh model, that has been extracted from a pre-operative 3-D CT data set. Furthermore, visualization without any 3-D object model is feasible as well. In this scenario, shown in Figure 6(b), landmark annotations, i.e. reconstructed pulmonary vein ostia or the coronary sinus, are used for orientation. The benefits of this approach are simplicity and no need for integration of pre-procedural or intra-procedural 3-D imaging. However, this approach offers only minimal anatomical information.

All of these visualization options allow to easily evaluate the distribution and quality of ablation points after the procedure. This information could be used to identify single ablation points or areas where occurrence of gaps is likely, either due to a lack of ablation points or poor lesion quality.

\section{DISCUSSION AND CONCLUSIONS}

We presented a novel tool for post-procedural ablation point evaluation of contact force characteristics to support $\mathrm{RF}$ ablation procedures. Classification thresholds for several contact force parameters have been estimated and applied to clinical data for evaluations. The main benefit of the proposed approach is the combined visualization of 3-D location and contact force characteristics for each created ablation lesion. This facilitates post-procedural verification of the ablation point distribution to detect and prevent the occurrence of gaps. This way, it may be possible to minimize the probability of AF recurrence. As of now, the thresholds used for color coding of the contact force characteristics have not been verified by clinical studies. Another aspect not covered in this work, is the use of anatomical information to dynamically adapt thresholds based on the specific location of the lesion. So far, these thresholds have only been estimated as averages over the whole procedure. 


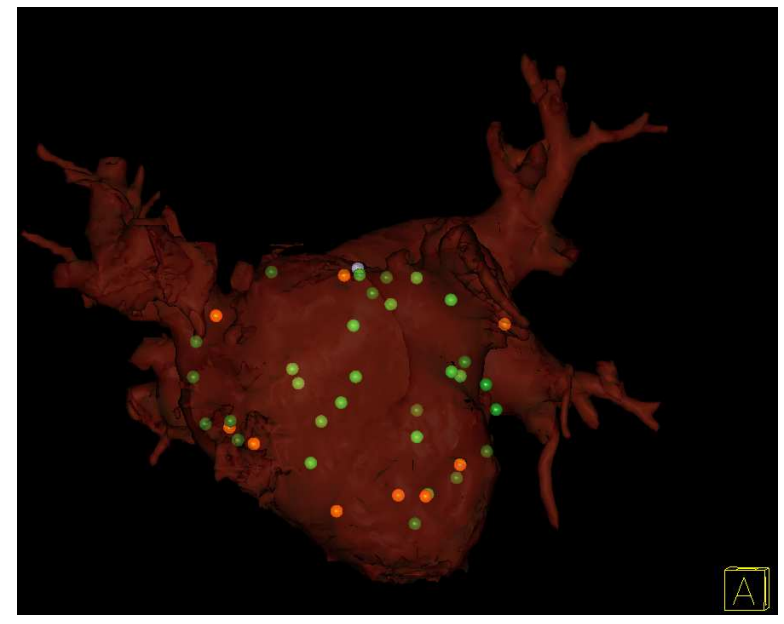

(a)

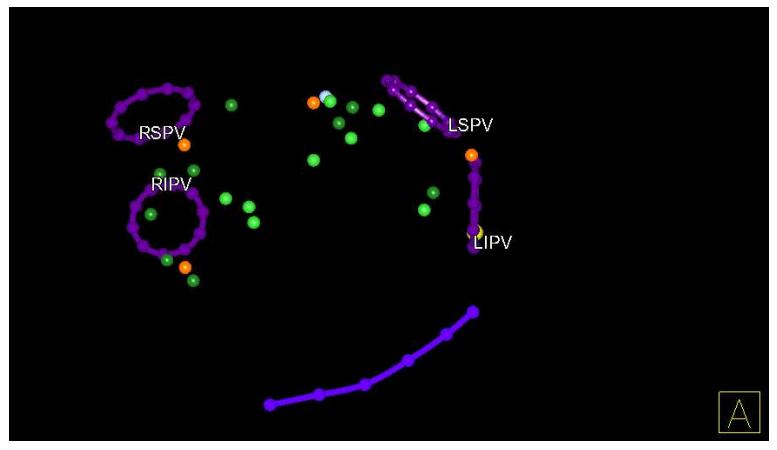

(b)

Figure 6. Visualization of color coded RF ablation points.

The information collected will be beneficial for physicians to document and verify ablation procedures. Going one step further, intra-procedural assessment of the lesion quality performed during a wait period might also help to reduce the number of procedures that have to be repeated due to recurrence of $\mathrm{AF} .{ }^{13}$

\section{ACKNOWLEDGMENT}

We thank Dr. Dipen Shah (University Hospitals of Geneva, Switzerland) for providing the clinical data. This work was supported by the German Federal Ministry of Education and Research (BMBF) in the context of the initiative Spitzencluster Medical Valley - Europäische Metropolregion Nürnberg, project grant Nos. 01EX1012A and 01EX1012E, respectively. Additional funding was provided by Siemens AG, Healthcare Sector.

\section{REFERENCES}

[1] C. Pappone, G. Vicedomini, G. Augello, F. Manguso, M. Saviano, M. Baldi, A. Petretta, L. Giannelli, Z. Calovic, V. Guluta, L. Tavazzi, and V. Santinelli, "Radiofrequency catheter ablation and antiarrhythmic drug therapy / clinical perspective," Circulation: Arrhythmia and Electrophysiology 4(6), 808-814 (2011).

[2] H. Oral, B. P. Knight, H. Tada, M. Özaydin, A. Chugh, S. Hassan, C. Scharf, S. W. Lai, R. Greenstein, F. Pelosi, S. A. Strickberger, and F. Morady, "Pulmonary vein isolation for paroxysmal and persistent atrial fibrillation," Circulation 105(9), 1077-1081 (2002).

[3] D. C. Shah, V. Y. Reddy, J. Kautzner, N. Saoudi, C. H. Siklódy, P. Jais, G. Hindricks, A. Yulzari, H. Lambert, P. Neuzil, and K.-H. Kuck, "Contact force during ablation predicts AF recurrence at 12 months," in Heart Rhythm Society, (2011).

[4] A. Thiagalingam, A. D'Avila, L. Foley, J. L. Guerrero, H. Lambert, G. Leo, J. N. Ruskin, and V. Y. Reddy, "Importance of catheter contact force during irrigated radiofrequency ablation: Evaluation in a porcine ex vivo model using a force-sensing catheter," Journal of Cardiovascular Electrophysiology 21(7), 806-811 (2010).

[5] K. Yokoyama, H. Nakagawa, D. Shah, H. Lambert, G. Leo, N. Aeby, A. Ikeda, J. Pitha, T. Sharma, R. Lazzara, et al., "Novel Contact Force Sensor Incorporated in Irrigated Radiofrequency Ablation Catheter Predicts Lesion Size and Incidence of Steam Pop and Thrombus," Circulation: Arrhythmia and Electrophysiology 1(5), 354 (2008).

[6] C. Piorkowski, H. Sih, P. Sommer, S. Miller, T. Gaspar, L. Teplitsky, and G. Hindricks, "First in human validation of impedance-based catheter tip-to-tissue contact assessment in the left atrium," Journal of Cardiovascular Electrophysiology 20(12), 1366-1373 (2009). 
[7] M. Koch, A. Langenkamp, A. Kiraly, A. Brost, N. Strobel, and J. Hornegger, "Navigation system with contact force assessment to guide pulmonary vein isolation procedures," in 23rd Conference of the Society for Medical Innovation and Technology, SMIT (2011).

[8] A. Brost, N. Strobel, L. Yatziv, W. Gilson, B. Meyer, J. Hornegger, J. Lewin, and F. Wacker, "Geometric Accuracy of 3-D X-Ray Image-Based Localization from Two C-Arm Views," in Workshop on Geometric Accuracy In Image Guided Interventions - MICCAI, 12-19 (2009).

[9] D. Shah, H. Lambert, H. Nakagawa, A. Langenkamp, N. Aeby, and G. Leo, "Area under the real-time contact force curve (force-time integral) predicts radiofrequency lesion size in an in vitro contractile model," Journal of Cardiovascular Electrophysiology 21(9), 1038-1043 (2010).

[10] S. M. Singh, E. K. Heist, D. M. Donaldson, R. M. Collins, J. Chevalier, T. Mela, J. N. Ruskin, and M. C. Mansour, "Image integration using intracardiac ultrasound to guide catheter ablation of atrial fibrillation," Heart Rhythm 5(11), 1548 - 1555 (2008).

[11] C. J. McGann, E. G. Kholmovski, R. S. Oakes, J. J. Blauer, M. Daccarett, N. Segerson, K. J. Airey, N. Akoum, E. Fish, T. J. Badger, E. V. DiBella, D. Parker, R. S. MacLeod, and N. F. Marrouche, "New magnetic resonance imaging-based method for defining the extent of left atrial wall injury after the ablation of atrial fibrillation," Journal of the American College of Cardiology 52(15), 1263 - 1271 (2008).

[12] V. Y. Reddy, "Low catheter-tissue contact force results in late PV reconnection - initial results from EFFICAS I," in Heart Rhythm Society, (2011). Talk.

[13] D. C. Shah, H. Sunthorn, H. Burri, and P. Gentil-Baron, "Does prolonged post PVI surveillance reduce PV conduction recurrence?," Heart Rhythm 7(5), 393 (2010). 